Article

\title{
Standard CMOS Fabrication of a Sensitive Fully Depleted Electrolyte-Insulator-Semiconductor Field Effect Transistor for Biosensor Applications
}

\author{
Gil Shalev, Ariel Cohen, Amihood Doron, Andrew Machauf, Moran Horesh, Udi Virobnik, \\ Daniela Ullien and Ilan Levy *
}

Intel Research Israel, Intel Electronics, Jerusalem 91031, Israel; E-Mails: gil.shalev@intel.com (G.S.); ariel.cohen@intel.com (A.C.); amihood.doron@intel.com (A.D.); andrew.machauf@intel.com (A.M.); moran.horesh@intel.com (M.H.); udi.virobnik@intel.com (U.V.);daniela.ullien@intel.com (D.U)

* Author to whom correspondence should be addressed; E-Mail: ilan.levy@intel.com; Tel.: +972-2589-7063; Fax: +972-2589-6342

Received: 25 March 2009; in revised form: 20 May 2009 / Accepted: 25 May 2009 /

Published: 4 June2009

\begin{abstract}
Microfabricated semiconductor devices are becoming increasingly relevant for detection of biological and chemical components. The integration of active biological materials together with sensitive transducers offers the possibility of generating highly sensitive, specific, selective and reliable biosensors. This paper presents the fabrication of a sensitive, fully depleted (FD), electrolyte-insulator-semiconductor field-effect transistor (EISFET) made with a silicon-on-insulator (SOI) wafer of a thin 10-30 nm active SOI layer. Initial results are presented for device operation in solutions and for bio-sensing. Here we report the first step towards a high volume manufacturing of a CMOS-based biosensor that will enable various types of applications including medical and enviro nmental sensing.
\end{abstract}

Keywords: EISFET; biosensors; CMOS; fully depleted

\section{Introduction}

In recent years, research and development of biosensors has received a great deal of attention since their extensive application potential is highly recognized in areas such as medical diagnostics and the 
food industry [1-4]. Biosensors are normally composed of two main components - the sensing device and the sensing molecule, i.e. chemical or biological recognition elements. As a functional hybrid system, the biggest challenge is to optimize this system to benefit from coupling of the unique features of the bio-recognition event with the sensitive signal recognition and amplification potential of a sensing device [5].

One of the major classes of silicon-based sensors uses a field-effect transistor (FET) as the transduction element for the analytical signals. In its basic configuration, the FET sensor is a fieldeffect transistor where the metal gate electrode is replaced by an ion-conducting solution and a reference electrode. This Electrolyte-Insulator-Semiconductor FET is commonly referred to as an EISFET. An inorganic dielectric material is used as the interface between the device and the solution, and its electrical response is sensitive to ion concentration in the solution [6-9]. This ion-sensitive field-effect transistor (ISFET) was first introduced by Bergveld in 1970 [10]. Eventually a membrane or other element can be added to the dielectric material to couple the biological components and to induce selectivity towards specific analytes [11]. Among various types of transducers used for biosensing, EISFET, along with its many inherent drawbacks is still one of the most investigated devices for electronic biosensing [12,13]. During the past 30 years, EISFET technology and applications have achieved a remarkable level of development [14]. FET-related devices have appeared with molecular selectivity (enzyme sensors [15], immunosensors [16] and DNA sensors [17]) and even with the ability of measuring complex biological receptors and cells [18]. Therefore, achieving an optimized sensor behavior in EISFETs usually requires the use of specific materials and device architectures.

The ability to detect biomolecular interactions is of extreme importance in medical diagnostics. Nevertheless, it also often requires single-use disposable sensors that would be fabricated in high volumes and will require a very low unitary cost. Much effort is being laid on nanowire-based FETs for biosensing. However, these nanowire-based devices suffer from very low manufacturing potential [19-21]. On the other hand, silicon microelectronic technology can provide a low cost manufacturing infrastructure for a high volume fabrication, but this requires the use of standard manufacturing process. Here we describe a standard CMOS manufacturing of an EISFET. The EISFET reported here comprises a thin conducting layer of $10-30 \mathrm{~nm}$ Silicon-On-Insulator (SOI) which implies that the active silicon is fully depleted (FD) for the given silicon doping. It was already demonstrated that FD EISFETs present enhanced electrical performance in terms of increased sensitivity to surface potential variations [22,23]. This sensitive device holds the potential application in medical diagnostics for biomarker analysis.

\section{Experimental}

\subsection{Device Fabrication}

6" Silicon-on-Insulator (SOI) wafers were used (SOITEC, Bernin, France). SOI layer and buriedoxide (BOX) thickness was $260 \mathrm{~nm}$ and $1,000 \mathrm{~nm}$, respectively, while SOI resistivity was $13-22 \Omega \mathrm{cm}$. Two types of devices were fabricated under the same process: FD EISFETs and metal-oxidesemiconductor FET (MOSFET) like devices that serve as test structures for process evaluation and 
electrical definition. Henceforth, these MOSFETs will be referred to as test structures. The fabrication of the devices was as follows: SOI layer was thinned down using oxidation and oxide removal. Several consecutive oxidation/oxide-removal steps took place in order to ensure a small thickness variation across the wafers. Eventually, several wafers with SOI thickness in the $10-30 \mathrm{~nm}$ range were fabricated with with-in-wafer SOI thickness variation not greater than $10 \%$. The SOI EISFET is fully depleted (FD) for the given SOI resistivity (doping) and SOI thickness. MESA-type isolation was used between the devices. Subsequent arsenic implant (15 keV/5 e14)) for the source and drain regions took place followed $100 \mathrm{~nm} \mathrm{SiO} 2$ PECVD for inter layer dielectric (ILD) and opening of the contacts. $\mathrm{Ti} / \mathrm{Al} / \mathrm{TiN}$ was sputtered and patterned for interconnection purposes followed by 4,500 $\AA$ passivation layer of PECVD nitride. The seed Ti/Au layer was sputtered followed by Au electroplating in the pad areas. The metal gate of the MOSFETs is located over a $100 \mathrm{~nm} \mathrm{PECVD} \mathrm{SiO}_{2}$ layer which is significantly thicker than the $30 \mathrm{~nm}$ LPCVD SiO$_{2}$ of the FD EISFETs. The last step of the process was the actual opening of the passivation above the FD EISFETs' active region. This was performed with dry etch followed by final wet etch in order to ensure no physical and/or electrical damage to the underlying active gate.

In the overall die layout (Figure 1), the central gold circle defines the sensing area, and the location of the sealing O-ring of the liquid flow-cell. The lower part contains the test structures zone. On the left side, a chemical window $(1.5 \mathrm{~mm} \times 1.5 \mathrm{~mm})$ is located that was used in order to perform detailed surface analysis (AFM and ellipsometry).

Figure 1. Schematic illustration of die layout $(17 \mathrm{~mm} \times 17 \mathrm{~mm})$.

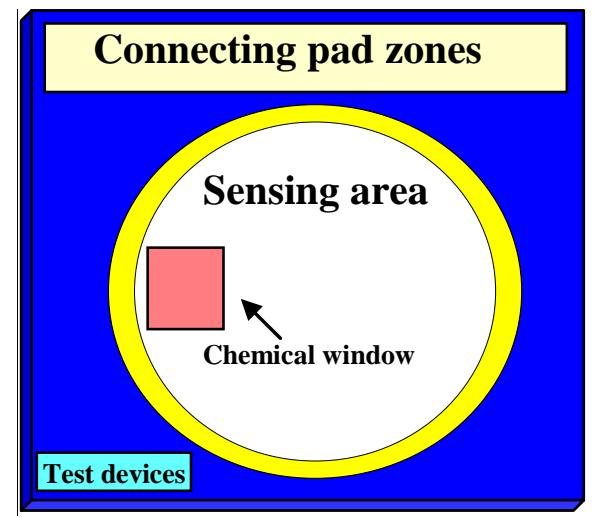

\subsection{Electronic Measurements}

I-V measurements were performed for both test structures and FD EISFETs. For the FD EISFETs, I-V measurements were performed both under dry and wet conditions. The electrical setting for both the test structures and the FD EISFETs wet measurements are presented in Figures 2A,B, respectively. In order to work in aqueous conditions we designed a unique liquid application apparatus - a flow cell (Figure 3). This unique design facilitated the work in aqueous enviro nments without the need for contact isolation. The liquids were retained within the O-ring gasket while the thumb screws were tightened against the probe-station chuck. The connecting pads were left out, providing easy approach for electrical testing (see also Figure 1). The apparatus included additional important features; ultralow sample volume $(30 \mu \mathrm{L})$, fast and convenient way of die replacement and black material to prevent 
light induced currents. A homemade $\mathrm{Ag} / \mathrm{AgCl}$ wire type reference electrode $\left(V_{\mathrm{REF}}\right)$ was used for the wet $\mathrm{I}-\mathrm{V}$ measurements that were performed with various solutions. I-V measurements of the test structures and the dry FD EISFETs were performed with an analog tester (Agilent - HP4062) and automatically probed by an Electroglass EG2010 probe.

Figure 2. Schematic cross-sections of A) test structure and B) EISFET. The gate oxide thickness of the test structure is $\sim 100 \mathrm{~nm}$, while the gate oxide thickness of the EISFET is $\sim 30$. Both devices are based on thin, 10 to $40 \mathrm{~nm}$ SOI layer. The source/drain regions are connected through metallization which in turn is protected by passivation layer. The distance between the drain and the source defines the channel length.
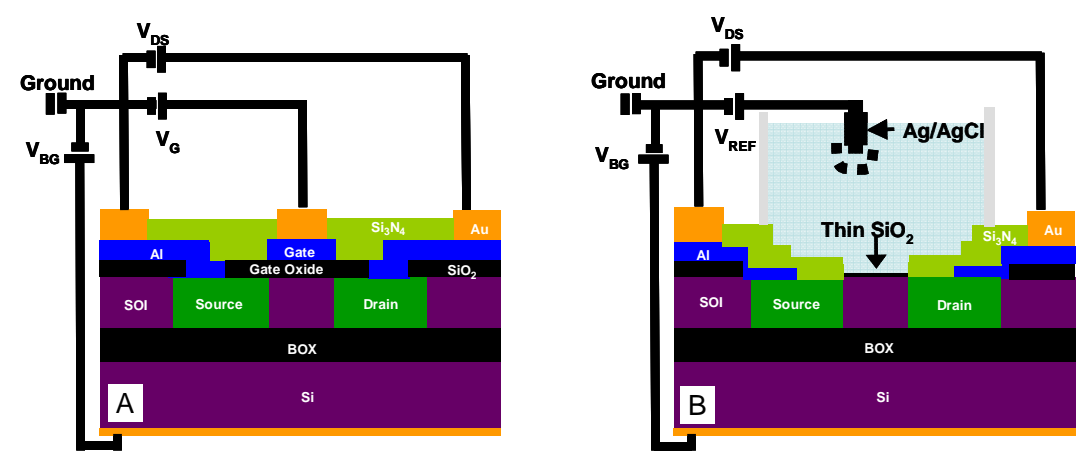

Figure 3. Liquid flow-cell design and manufacture. Left: cross-section illustrating the inlet and the outlet tubing entrances. The silicon die is located at the bottom of the flow-cell held tight using 4 thumbscrews (middle picture). The O-ring holds the die in close proximity with the flow-cell, maintaining all liquids within the die 'wet' area far away from the contact pads. The right picture is the actual flow-cell with its reference electrode entrance. This flow-cell design includes the following unique features: low sample volume $(30 \mu \mathrm{L})$, ease of sample replacement, light protecting and chemically stable material.
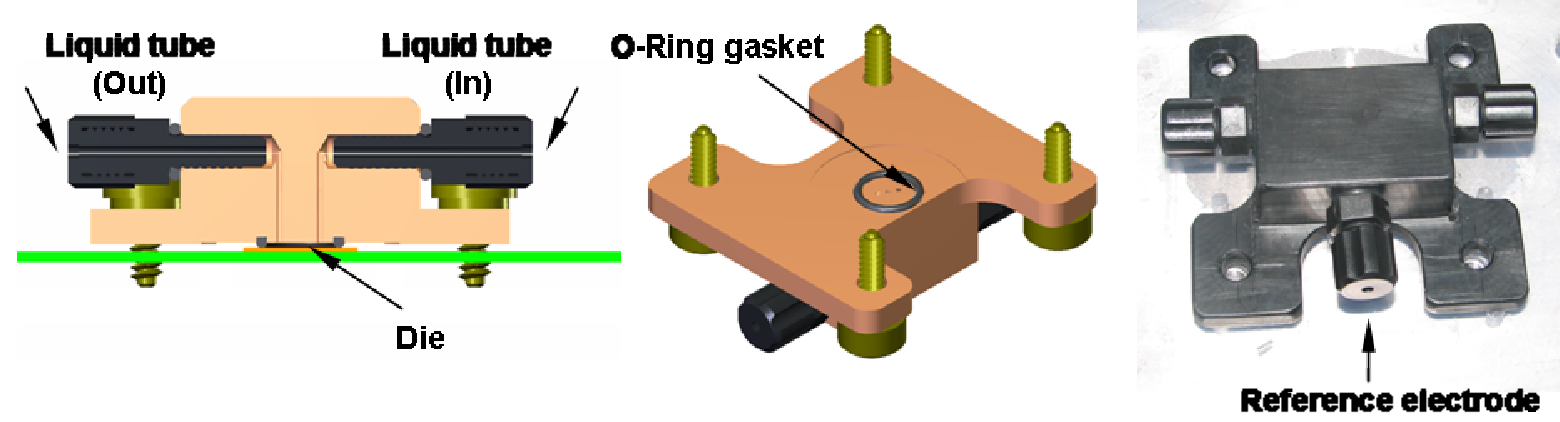

\subsection{Device Functionalization}

After a brief dip in distilled water (18 Mohm) and drying under streaming $\mathrm{N}_{2}$, devices were activated by G-1000 Oxygen down stream plasma (Yield Engineering Systems Inc., San Jose, CA, USA), and immediately modified for the specified time in ethanol:water (95:5) solution containing 1\%(v/v) aminopropyltrimethoxysilane (APTMS, Gelest Inc., Morrisville, PA, USA). The devices were then washed with ethanol, dried over $\mathrm{N}_{2}$, and kept desiccated in closed vial for further measurements (not longer than 24 hours). Surface response to various treatments (Figure 10B) was monitored as 
previously described [23]. Briefly, devices were activated using ultraviolet ozone cleaning system (UVOCS, T10X10, Montgomeryville, PA) followed by APTMS monolayer formation. The sample was then immersed in strong acid, dried, and measured, followed by the same process but with strong base.

\subsection{Contact Angle Analysis}

A contact angle meter (Ramé-Hart instrument co. Netcong, NJ, USA) was used to measure the static water contact angle of the films. Contact angles of $4 \mu \mathrm{L}$ water drops were measured according to a standard method [24] during surface modification at room temperature. The reported results are the average of six different samples taken at three different spots of a given sample.

\section{5. pH Measurements}

Following 4 min of APTMS activation (described in a previous section) the devices were inserted into the custom-made flow-cell for $\mathrm{pH}$ measurements. The flow-cell was then washed with $2 \mathrm{~mL}$ of 10 $\mathrm{mM}$ phosphate buffer (PB) with $\mathrm{pH}$ value of 6.2 in order to initiate the experiment. The device was subjected to $5 \mathrm{~mL}$ of $\mathrm{PB}$ solution with different $\mathrm{pH}$ values $(\mathrm{pH}: 6.2,6.7$ and 8.2). The source-drain current $\left(I_{\mathrm{DS}}\right)$ was measured continuously and the modulation of $I_{\mathrm{DS}}$ with the various $\mathrm{pH}$ levels was recorded. In order to translate the $I_{\mathrm{DS}}$ modulation into $\mathrm{pH}$ sensitivity in terms of $\mathrm{mV} / \mathrm{dec}$, a $10 \mathrm{mV}$ calibration pulses were given at the relevant $\mathrm{pH}$ levels in order to extract the FD EISFET gain $\left(g_{m}\right)$. The time-based $\mathrm{pH}$ measurements were performed in the linear region with $V_{\mathrm{DS}}=1 \mathrm{~V}, V_{\mathrm{REF}}=1.5 \mathrm{~V}$ and $V_{\mathrm{Gb}}=50 \mathrm{~V}$.

\subsection{AFM Surface Analysis}

The topographies of the thin films were investigated by AFM (Solver P47 PRO Scanning Probe Microscope). AFM was operated in contact mode in air and at room temperature. Images were acquired at a scan rate of $1.5 \mathrm{~Hz}$ with a silicon cantilever (Ultralever $06 \mathrm{~B}$, PSI, USA), and the scan size used was $1 \mu \mathrm{m} \times 1 \mu \mathrm{m}$.

\section{Results and Discussion}

\subsection{Fabrication}

Figures 2A and 2B depict schematic cross-sections of the test structure and FD EISFET, respectively, with their basic layers. The source/drain regions are connected through metallization which in turn is protected by passivation layer as can be seen in Figure 4. The role of the passivation layer is to avoid shorts and to protect the metallization from chemical solution attack. Due to the shallow SOI layer the drain and source regions extend vertically down to the buried oxide. The cleanroom process steps of the chemical window are identical to the FD EISFET active area (gate). Figures 4A and B illustrates representative SEM cross-section where the sensing area is located in the middle 
surrounded by drain/source regions connected to metallization. The metallization layer is covered by the passivation nitride layer. TEM analysis (Figure 4C) demonstrates the various layers in the device including a device with a thin $\sim 10 \mathrm{~nm}$ SOI layer. The last process step (opening the active gate area) was monitored using AFM roughness analysis (Figure 5) indicating that this step did not resulted with a significant roughness increase maintaining RMS roughness of $0.62 \mathrm{~nm}$.

Figure 4. SEM and TEM images of device cross-sections. (A) A tilted top view of the sliced device. (B) Side view cross-section of the same device. Sensing area is shown in the middle surrounded by drain/source regions connected to metallization layer. (C) Side view TEM cross section showing relative thickness of the different layers that are illustrated in Figure 2.
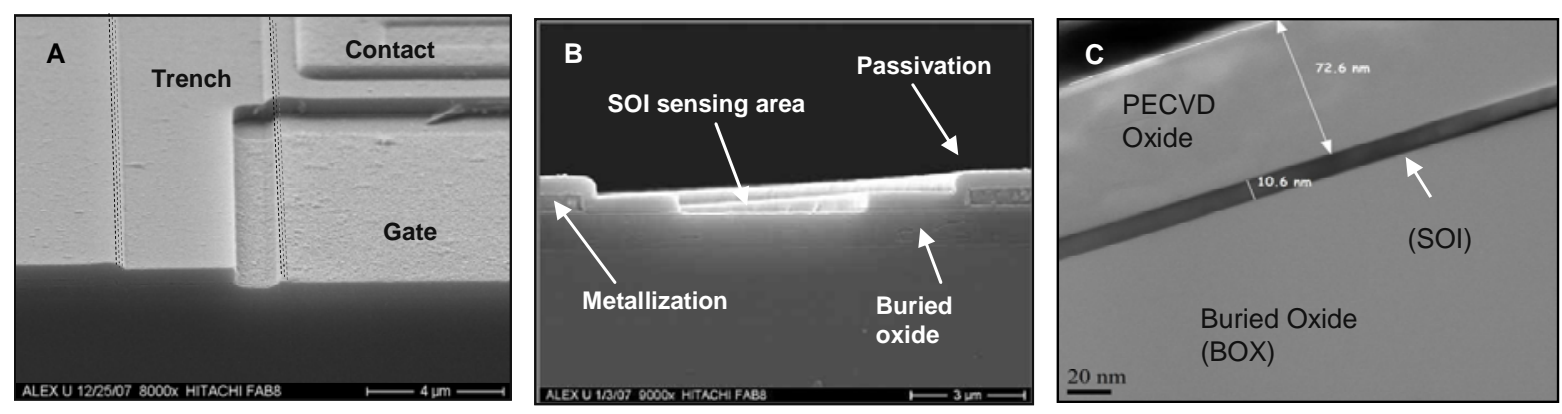

Figure 5. AFM tapping mode topographic image of the active gate area.

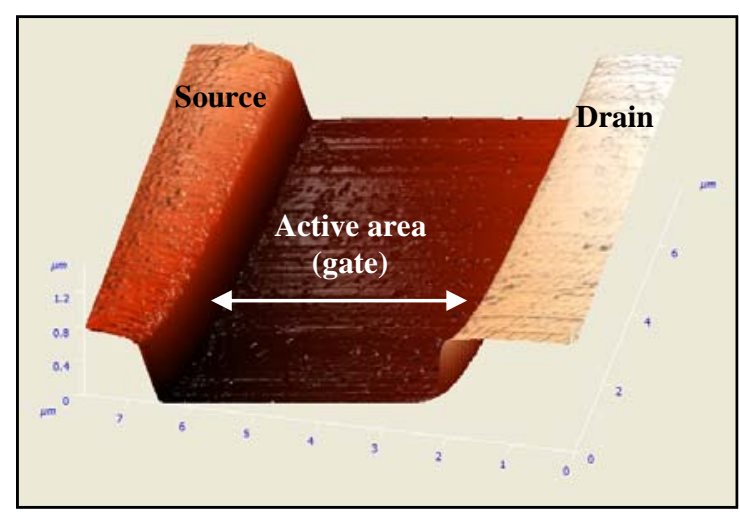

Figure 6. I-V characterization of a typical test structure. $I_{\mathrm{DS}}$ versus $V_{\mathrm{GS}}$ of a depletion type (n-type) device fabricated with a $10 \mathrm{~nm}$ SOI layer.

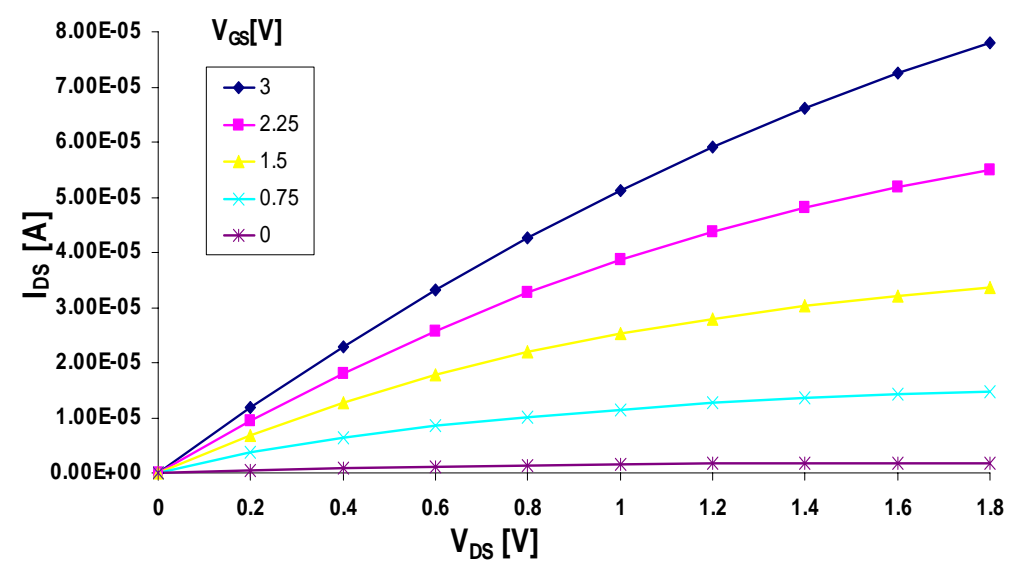


Test structures were fabricated in order to monitor and evaluate the performance of the clean room fabrication, and also provide means by which standard and automatic CMOS electrical testing (ETEST) can be performed. Figure 6 presents the I-V curve of an n-type rectangular-shaped test structure with a channel width and length of $20 \mu \mathrm{m}$ and $5 \mu \mathrm{m}$, respectively. The back-gate $\left(V_{\mathrm{Gb}}\right)$ voltage was set to $0 \mathrm{~V}$, the drain-source voltage $\left(V_{\mathrm{DS}}\right)$ was swapped between 0 and $1.8 \mathrm{~V}$ with $0.2 \mathrm{~V}$ intervals, and the front gate voltage was swapped between $0 \mathrm{~V}$ and $3 \mathrm{~V}$ with $0.75 \mathrm{~V}$ intervals. It is demonstrated that the threshold voltage $\left(V_{\mathrm{T}}\right)$ and transconductance $\left(g_{\mathrm{m}}\right)$ are $\sim 0 \mathrm{~V}$ and $\sim 29 \mu \mathrm{A} / \mathrm{V}$ respectively. Accordingly, the device is a depletion type device with a relatively high $g_{\mathrm{m}}$. The test structure performance indicated that the thin SOI layer retains its single crystal properties throughout processing.

\subsection{Electrical Testing}

I-V electrical characterization of the FD EISFET was performed pre surface modification. Representative $I_{\mathrm{DS}}$ vs. $\mathrm{Ag} / \mathrm{AgCl}$ reference electrode voltage $\left(V_{\mathrm{REF}}\right)$ at $\mathrm{pH} 7$ for different back gate voltage $\left(V_{\mathrm{Gb}}\right)$ are presented in Figure 7 . The width $(\mathrm{W})$ and length $(\mathrm{L})$ of the measured FD EISFET are $100 \mu$ and $10 \mu$, respectively. Note that higher values of $V_{\mathrm{Gb}}$ imply reduction in front threshold voltage $\left(V_{\mathrm{Tf}}\right)$. This $V_{\mathrm{Tf}}$ dependency upon $V_{\mathrm{Gb}}$ is a manifestation of the existing charge coupling between the front and back interfaces in FD SOI. The continuous decrease in $V_{\mathrm{Tf}}$ for increasing $V_{\mathrm{Gb}}$ reflects the transition of the back interface from accumulation into inversion. More specifically, the more depleted the back interface, the lower is $V_{\mathrm{Tf}}$. For inverted back interface, $V_{\mathrm{Tf}}$ is lowest and constant, and for accumulated back interface $V_{\mathrm{Tf}}$ is highest and constant [22,25]. Note that inversion and accumulation of the back interface are not apparent in the present case. A more comprehensive descriptions of the basic properties of the sensor device can be found in our previous publications $[22,23,26]$.

Figure 7. $I_{\mathrm{DS}}$ vs. $V_{\mathrm{REF}}$ of an EISFET for various values of $V_{\mathrm{Gb}}$. Note the shift in front threshold voltage with increasing $V_{\mathrm{Gb}}\left(V_{\mathrm{DS}}=0.5 \mathrm{~V}\right)$.

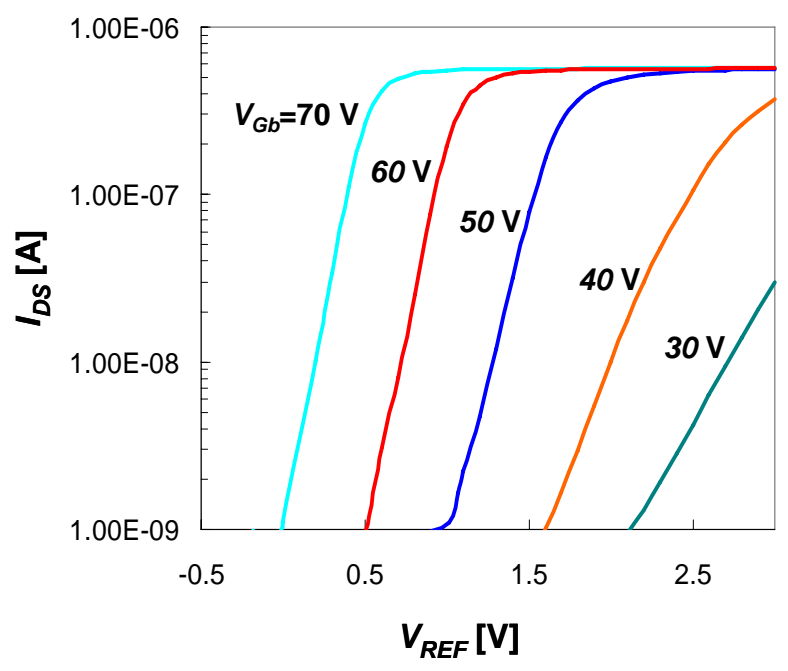




\subsection{Activation}

Choosing optimal activation conditions are of significant importance for FET-based biosensors. Parameters like surface morphology and self-assembly film activity depends on many factors such as reagent concentration, type of solvent and water percentage. The most common and successful route for the deposition of self assembled monolayer (SAM) on Si substrates is through silanization process. In this process, SAMs are formed spontaneously by immersing the $\mathrm{OH}$-terminated $\mathrm{SiOx} / \mathrm{Si}$ substrates into an active solution. The mechanism of SAM formation process is well established, which is known to take place in four steps: 1) physical adsorption onto the hydrated silicon surface, 2) hydrolysis of the silane head-groups $\left(\mathrm{SiX}_{3}\right)$ in the presence of the adsorbed water layer on the surface into highly polar silanetriol $\left.\mathrm{Si}(\mathrm{OH})_{3}, 3\right)$ covalent bonds formation of the silanetriol with the silanol groups $(\mathrm{Si}-\mathrm{OH})$ on substrate surface, and 4) self-organization of the monolayer driven by van der Waals interactions among the linear alkyl-chains into a packed dense layer [27-29]. During the initial stage (short incubation times), only a few molecules will adsorbed on the substrate surface and it is expected that the monolayer will be in a disordered (or liquid) state. However, at longer incubation times, the surface coverage eventually reaches the point where a well-ordered and compact monolayer is obtained. It was also demonstrated that the growth kinetics depends on the alkyl-chain length that is a result of chain length dependent chemisorption and diffusion rates of molecules on the substrate surface, which are known to decrease with increasing chain length [30].

Figure 8. AFM tapping mode topographic images of modified surfaces. Surface analysis was done following $20 \mathrm{~min}$ UVOCS and $2 \mathrm{~min}$ (A), or $10 \mathrm{~min}$ (B), APTMS (aminopropyltrimethoxysilane) monolayer formation. Topographical scans were done in non contact mode with $\mathrm{Si}_{3} \mathrm{~N}_{4}$ pyramidal tips with tip curvature of $\sim 15 \mathrm{~nm}$, force constant (k) $\sim 0.5 \mathrm{~N} / \mathrm{m}$, and with scan rate of $0.5 \mathrm{~Hz}$. The data obtained for A was: RMS roughness: $0.3 \mathrm{~nm}$, average height: $1.07 \mathrm{~nm}$, Min/Max: $0.77 \mathrm{~nm}$, and for B: RMS roughness: $0.31 \mathrm{~nm}$, average height: $0.94 \mathrm{~nm}$, Min/Max: $2.52 \mathrm{~nm}$. (C) RMS roughness comparison analysis for different pre-activation conditions.
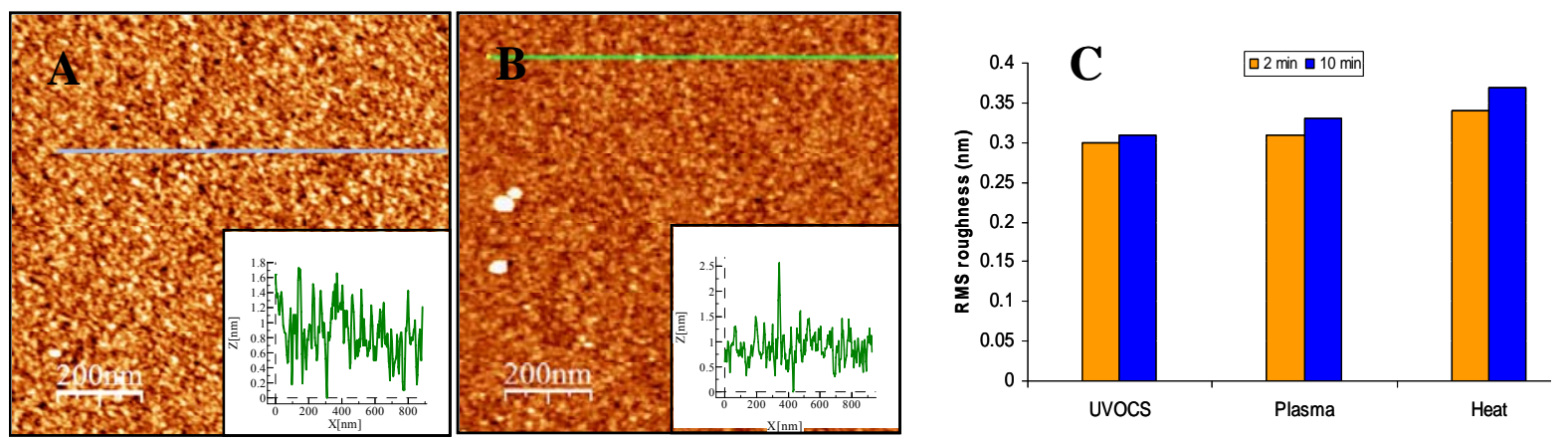

Our data presented in Figures 8 and 9 supports this model, demonstrating that by changing preactivation conditions, modification time and reagent mixtures, we could control saturation curves in single degree resolution. Typical AFM images of the samples prepared using fixed APTMS concentration in various incubation times had demonstrated a height profile with a uniform coverage of the substrate with a surface roughness $<1 \AA$ (Figures $8 \mathrm{~A}$ and B). We also demonstrated that the pre- 
activation conditions have small effect on the RMS roughness (Figure 8C). This analysis verify our procedure to be one that results with very smooth film with low RMS roughness which is similar to the roughness of the silicon surface itself. Contact angle provided a complementary method for SAM characterization since it is very sensitive to the differences between the hydrophobic natures of the alkyl chain versus the hydrophilic nature of the animated head group.

Figure 9. Correlation between contact angle and APTMS modification time (1, 3, 5 and 10 min). Working conditions chosen for device modification are indicated by dashed arrow. This figure illustrates the graduate changes (increase) in contact angle as the monolayer (APTMS) deposition time increases.

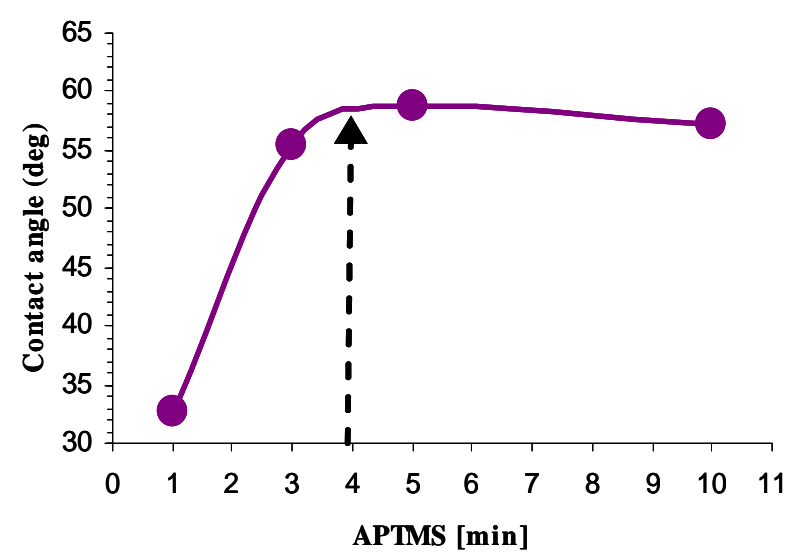

The measured DI water contact angles indicated the direct correlation between incubation time and contact angle up to a level of a uniform layer (Figure 9). It is important to note that the reported water contact angles for $\mathrm{NH}_{2}$-terminated surfaces are highly variable and data between 15 and 68 are reported [31,32]. This discrepancy is probably a result of the high activity of $\mathrm{NH}_{2}$ head group adsorbing impurities from the atmosphere in the time duration between SAM formation and contact angle measurements [33,34]. We have concluded that shorter incubation time resulted with nonuniform monolayers whereas longer time duration resulted with better surface coverage and a more uniform layer. The chosen working conditions represent "mid-point" of full coverage APTMS self assembly similar to what is reported in literature $\left(\phi a \sim 42^{\circ}\right.$ to $\left.68^{\circ}\right)$ (Figure 9) [35].

\section{4. $p H$ Sensing}

We have used our flow-cell apparatus in order to monitor on-line device response to $\mathrm{pH}$ alteration presenting the results over time (Figure 10A). The drain current increased or decreased depending on the $\mathrm{pH}$ value. The APTMS activated, $\mathrm{n}$-channel device demonstrates decreasing $I_{\mathrm{DS}}$ for increasing $\mathrm{pH}$ values, as expected. A pH sensitivity of $40 \mathrm{mV} / \mathrm{pH}$ is recorded which is lower than the theoretical value for silicon oxide in this $\mathrm{pH}$ range $(47 \mathrm{mV} / \mathrm{dec}$ for $\mathrm{pH} 6$ and $51 \mathrm{mV} / \mathrm{dec}$ for $\mathrm{pH}$ 8). Since the presented data is of APTMS activated surfaces, we anticipated that the theoretical value will not be obtained. Furthermore, considering the mixed amphoteric surface, our results indicate on very sensitive devices. In addition, these devices demonstrated stable $\mathrm{pH}$ sensitivity in electrolyte solution or in dry condition over time up to several months (data is not presented). 
Figure 10. (A) pH sensitivity of APTMS activated depletion type (n-type) transistor with a $10 \mathrm{~nm}$ thin Si layer. $I_{\mathrm{DS}}$ vs. time was taken for different $\mathrm{pH}$ values (the working point was set to the linear region at: $\left.V_{\mathrm{DS}}=1 \mathrm{~V}, V_{\mathrm{REF}}=1.5 \mathrm{~V}, V_{\mathrm{Gb}}=50 \mathrm{~V}\right)$. In order to determine $\mathrm{pH}$ sensitivity, the $I_{\mathrm{DS}}$ current was divided by $g_{\mathrm{m}}$ (calculated using calibration pulse). (B) Demonstrating device performance following APTMS activation. $V_{\mathrm{Gb}}$ was held constant at $50 \mathrm{~V}$. The base line is the I-V curve for device covered with activated native oxide (1). Following APTMS monolayer formation, a significant increase in currents can be detected (2). Incubating the device in strong acid (APTMS head group protonation) resulted with additional current boost (3). Consequently, head groups de-protonation using strong base, resulted in current decrease (4).
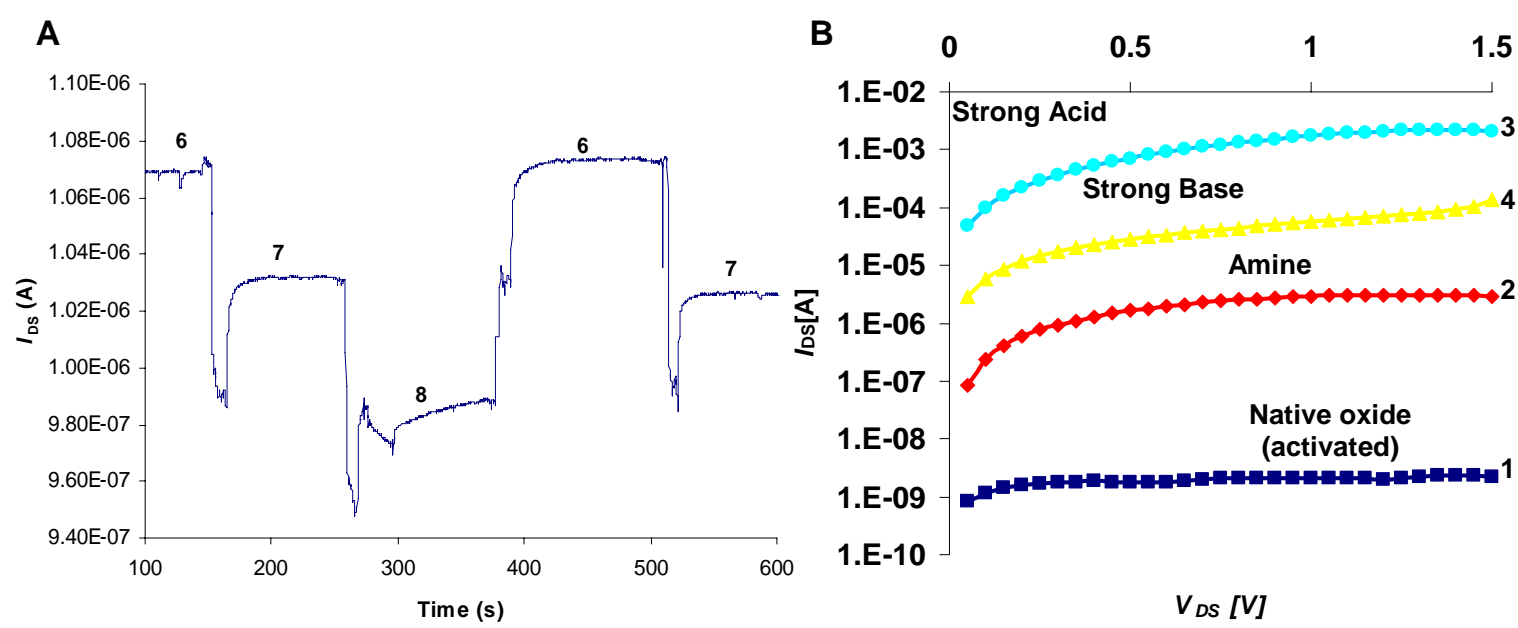

The $\mathrm{pH}$ sensitivity of amphoteric surfaces depends upon the intrinsic buffer capacity of the surface and the capacitance of the double layer created at the dielectric/solution interface. The intrinsic buffer capacity is described by the site-dissociation model that attributes the processes of protonation and deprotonation of titratable surface groups (e.g. amphoteric $\mathrm{OH}$ groups on $\mathrm{SiO}_{2}$ gate dielectric). The capacitance of the double layer is a function of the salt concentration and the surface potential [36]. Protonation/deprotonation processes are the key factors dominating device $\mathrm{pH}$ sensitivity through its significant impact on the electrochemical characteristics of amphoteric groups. In the present case, the mixed interface is composed of two amphoteric groups: the $-\mathrm{SiOH}$ with $\mathrm{pKa}$ value of 6.8 and $-\mathrm{NH}_{2}$ with pKa value of $9-10$ (attributed to APTMS amino group). Therefore it is anticipated that the interface is fully protonated at $\mathrm{pH}$ in the range of 6.8 to 9 [37-39]. The observed linear response (Figure 10A) can be attributed to an approximately linear change in the total surface charge density (versus $\mathrm{pH}$ ) because of the combined acid and base behavior of both surface groups [40]. In order to further illustrate this phenomenon we plotted device I/V curves following alteration of acidic and basic treatments (Figure 10B). As a base line we measured device performance following surface activation. The next step was APTMS monolayer modification that resulted with significant increase in drain current that can be attributed to positive charge accumulation. Protonation of the amine head group under acidic condition (transition from $-\mathrm{NH}_{2}$ to $-\mathrm{NH}_{3}{ }^{+}$) resulted with further accumulation of positive charge on the surface. Deprotonation of the amine group under basic condition resulted with decreased drain currents. 


\subsection{Sensing Biomolecular Interactions}

To investigate biomolecular interaction monitoring, we functionalized the gate surface with biotin and studied the well-known binding of biotin to streptavidin (Figure 11). The measurement was taken at the subthreshold regime with $V_{\mathrm{Gb}}=50 \mathrm{~V}, V_{\mathrm{DS}}=50 \mathrm{mV}$ and $V_{\mathrm{REF}}=1 \mathrm{~V}$, in $50 \mathrm{mM}$ phosphate buffer $\mathrm{pH}$ 7. Our measurements demonstrate a clear signal following the addition of $20 \mathrm{~nm}$ streptavidin. The observed decreased currents are consistent with binding of a negatively charged species to the activated gate surface, and the fact that streptavidin is a negatively charged molecule at the current working conditions (pI: 5.5). In addition, the stable signal is the result of the nearly covalent biotinstreptavidin binding $\left(\mathrm{Ka} \sim 10^{-15} \mathrm{M}^{-1}\right)$ [41]. The proposed mechanism for the sensing activity is that linking negatively charged molecule to the active gate area is equivalent to negative gate biasing resulting with changed surface potential that eventually modulate channel currents. The charge coupling between the front and back interfaces in FD EISFET and the subsequent ability to optimize the sensitivity of FD EISFET to surface potential variations was already demonstrated [22,23]. The current label-free measurement was executed at the subthreshold regime where the FD EISFET sensitivity is greater for higher $V_{\mathrm{GB}}$. Finally, drift measurements of this system was in the range of $\sim 3$ $\mathrm{mV} / \mathrm{h}^{-1}$, which is consistent with drift values cited in current literature [42]. As the length of measurement did not exceed $15 \mathrm{~min}$, the effect of drift current is negligible in comparison with the current increase due to strepavidin detection.

Figure 11. First label-free results from bio-sensing device based on the FD EISFET platform. The first structure analyzed for analyte detection was tested on a biotinstreptavidin model system. Results indicate that we can detect analyte (20 nm streptavidin) interacting with the capture molecule (biotin).

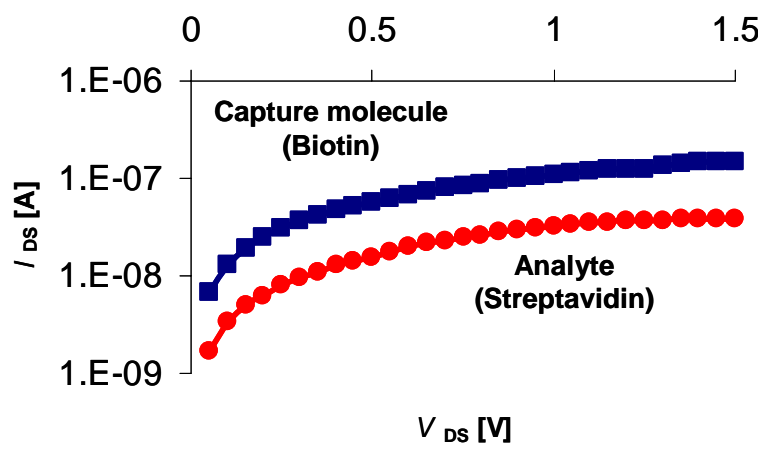

\section{Conclusions}

Here we present the first step into the fabrication of a low cost sensitive device with potential future application for bio-sensing. Successful exploitation of this exciting possibility is critically dependent on a high volume manufacturing potential that will result in a low unit cost. We expect that the high $g_{\mathrm{m}}$ values and the high $\mathrm{pH}$ sensitivity will lay the foundations for the detections of diverse biomolecular interactions. 


\section{References}

1. Pejcic, B.; De Marco, R.; Parkinson, G. The role of biosensors in the detection of emerging infectious diseases. Analyst 2006, 131, 1079-1090.

2. Rasooly, A.; Herold, K.E. Biosensors for the analysis of food and waterborne pathogens and their toxins. J. AOAC Int. 2006, 89, 873-883.

3. Soper, S.A.; Brown, K.; Ellington, A.; Frazier, B.; Garcia-Manero, G.; Gau, V.; Gutmang, S.I.; Hayes, D.F.; Brenda, K.; Landers, J.L.; Larson, D.; Ligler, F.; Majumdar, A.; Mascini, M.; Nolte, D.; Rosenzweig, Z.; Wang, J.; Wilson, D. Point-of-care biosensor systems for cancer diagnostics/prognostics. Biosens. Bioelectron. 2006, 21, 1932-1942.

4. Yang, C.H.; Liao, Y.W. An ISFET interface circuitry for biomedical applications. In Proceedings of IEEE Conference on Electron Devices and Solid-State Circuits, Tainan,Taiwan, December 2022, 2007; pp.1083-1086.

5. Killard, A.J.; Smyth, M.R. Creatinine biosensors: principles and designs. Trends Biotechnol. 2000, 18, 433-437.

6. Xu, J.J.; Luo, X.L.; Chen, H.Y. Analytical aspects of FET-based biosensors. Front. Biosci. 2005, 10, 420-430.

7. Machauf, A.; Cohen, A.; Doron, A.; Beraha, M.; Weinfeld, B.; Levy, I. Fabrication of a sensitive Field Effect Device (FED) for biosensor application. In Proceedings of ISIE 2007, Vigo, Spain, June 4-7, 2007; pp. 2820-2823.

8. Kal, S.; Bhanu Priya, V. Design and modeling of ISFET for $\mathrm{pH}$ sensing. In Proceedings of TENCON 2007, Taipei, Taiwan, October 30 - November 2, 2007; pp. 1-4.

9. Shinwari, M.W.; Deen, M.J.; Landheer, D. Study of the electrolyte-insulator-semiconductor fieldeffect transistor (EISFET) with applications in biosensor design. Microelectron. Reliab. 2007, 47, 2025-2057.

10. Bergveld, P. Development of an ion-sensitive solid-state device for neurophysiological measurements. IEEE Trans. Biomed. Eng. 1970, BME-17, 70-71.

11. Pan, T.M.; Liao, K.M.; Yen, L.C.; Hsieh, Y.Y.; Chen, Y.Z. Effects of oxygen content on the structural and sensing properties of Y203 sensing membrane for pH-ISFET application. In Proceedings of International Semiconductor Device Research Symposium, Maryland, USA, December 12-14, 2007; pp. 1-2.

12. Bergveld, P. Thirty years of ISFETOLOGY. What happened in the past 30 years and what may happen in the next 30 years. Sens. Actuat. B 2003, 88, 1-20.

13. Yuqing, M.; Jianguo, G.; Jianrong, C. Ion sensitive field effect transducer-based biosensors. Biotechnol. Adv. 2003, 21, 527-534.

14. Schoning, M.J.; Poghossian, A. Recent advances in biologically sensitive field-effect transistors (BioFETs). Analyst. 2002, 127, 1137-1151.

15. Park, K.; Choi, S.; Lee, M.; Sohn, B.; Choi, S. ISFET glucose sensor system with fast recovery characteristics by employing electrolysis. Sens Actuat. B 2002, 83, 90-97.

16. Starodub, N.F.; Dzantiev, B.B.; Starodub, V.M.; Zherdev, A.V. Immunosensor for the determination of the herbicide simazine based on an ion-selective field-effect transistor. Anal. Chim. Acta. 2000, 424, 37-43. 
17. Estrela, P.; Migliorato, P.; Takiguchi, H.; Fukushima, H.; Nebashi, S. Electrical detection of biomolecular interactions with metal-insulator-semiconductor diodes. Biosens. Bioelectron. 2005, 20, 1580-1586.

18. Offenhausser, A.; Knoll, W. Cell-transistor hybrid systems and their potential applications. Trends Biotechnol. 2001, 19, 62-66.

19. Nair, P.R.; Alam, M.A. Design considerations of silicon nanowire biosensors. IEEE Trans. Electron. Dev. 2007, 54, 3400-3408.

20. Thelander, C.; Fröbergfroberg, L.E.; Rehnstedt, C.; Samuelson, L.; Wernersson, L.E. Vertical enhancement-mode InAs nanowire field-effect transistor with 50- nm wrap gate. IEEE Electron. Dev. Lett. 2008, 29, 206-208.

21. Chen, Z.; Farmer, D.; Xu, S.; Gordon, R.; Avouris, P.; Appenzeller, J. Externally assembled gateall-around carbon nanotube field-effect transistor. IEEE Electron. Dev. Lett. 2008, 29, 183-185.

22. Shalev, G.; Doron, A.; Virobnik, U.; Cohen, A.; Sanhedrai, Y.; Levy, I. Gain optimization in ISFET based sensor with fully depleted silicon-on-insulator. Appl. Phys. Lett. 2008, 93, 1-3.

23. Shaya, O.; Shaked, M.; Doron, A.; Cohen, A.; Levy, I.; and Rosenwaks, Y. Distinguishing between dipoles and field effects in molecular gated transistors. App. Phys. Lett. 2008, 93, 043509, $1-3$.

24. Adamson, A. Physical Chemistry of Surfaces; John Wiley \& Sons: New York, NY, USA, 1982.

25. Lim, H.K.; Fossum, J.G. Threshold voltage of thin-film siliconon insulator (SOI) MOSFET's. IEEE Trans. Electron. Dev.1983, ED-30, 1244-1251.

26. Shaya, O.; Shaked, M.; Usherenko, Y.; Halperin, E.; Shalev, G.; Doron, A.; Levy, I.; Rosenwaks, Y. Tracing the mechanism of molecular gated transistors. J. Phys. Chem. 2009, 113, 6163-6168.

27. Aswal, D.K.; Lenfant, S.; Guerin, D.; Yakhmi, J.V.; Vuillaume, D. Self assembled monolayers on silicon for molecular electronics. Anal. Chim. Acta. 2006, 568, 84-108.

28. Brzoska, J.B.; Azouz, I.B.; Rondelez, F. Silanization of Solid Substrates: A step toward reproducibility. Langmuir 1994, 10, 4367-4373.

29. Wasserman, S.R.; Tao, Y.T.; Whitesides, G.M. Structure and reactivity of alkylsiloxane monolayers formed by reaction of alkyltrichlorosilanes on silicon substrates. Langmuir 1989, 5, 1074-1087.

30. Schwartz, D.K.; Steinberg, S.; Israelachvili, J.; Zasadzinski, J.A.N. Growth of a self-assembled monolayer by fractal aggregation. Phys. Rev. Lett. 1992, 69, 3354-3357.

31. Denoyel, R.; Glez, J.C.; Trens, P. Grafting $\gamma$-aminopropyl triethoxysilane onto silica: consequence on polyacrylic acid adsorption. Colloid Surf. A. 2002, 197, 213-223.

32. Horr, T.J.; Arora, P.S. Determination of the acid-base properties for 3-amino, 3-chloro and 3mercaptopropyltrimethoxysilane coatings on silica surfaces by XPS. Colloid Surf. A. 1997, 126, 113-121.

33. Allen, G.C.; Sorbello, F.; Altavilla, C.; Castorina, A.; Ciliberto, E. Macro-, micro- and nanoinvestigations on 3-aminopropyltrimethoxysilane self-assembly-monolayers. Thin Solid Films 2005, 483, 306-311.

34. Petri, D.F.; Wenz, G.; Schunk, P.; Schimmel, T. An improved method for the assembly of aminoterminated monolayers on $\mathrm{SiO}_{2}$ and the vapor deposition of gold layers. Langmuir 1999, 15, $4520-4523$. 
35. Siqueira Petri, D.F.; Wenz, G.; Schunk, P.; Schimmel, T. An improved method for the assembly of amino-terminated monolayers on $\mathrm{SiO}_{2}$ and the vapor deposition of gold layers. Langmuir 1999, 15, 4520-4523.

36. van Hal, R.E.G.; Eijkel, J.C.T.; Bergveld, P. A general model to describe the electrostatic potential at electrolyte oxide interfaces. Adv. Coll. Interf. Sci. 1996, 69, 31-62.

37. Iler, R.K. The Chemistry of Silica; Wiley: New York, NY, USA, 1979.

38. Notsu, H.; Fukazawa, T.; Tatsuma, T.; Tryk, D.A.; Fujiwara,Y. Hydroxyl groups on boron-doped diamond electrodes and their modification with a silane coupling agent. Electrochem. Solid-State Lett. 2001, 4, H1-H3.

39. Song, K.S.; Nakamura, Y.; Sasaki, Y.; Degawa, M.; Yang, J.H.; Kawarada, H. pH-sensitive diamond field-effect transistors (FETs) with directly aminated channel surface. Anal. Chim. Acta. 2006, 573, 3-8.

40. Cui,Y.; Wei, Q.; Park, H.; Lieber, C.M. Nanowire Nanosensors for highly sensitive and selective detection of biological and chemical species. Science 2001, 293, 1289-1292.

41. Wilchek, M.; Bayer, E.A. Biotin-binding proteins: overview and prospects. Methods Enzymol. 1990, 184, 49-51.

42. Shahriar J. An analytical technique for counteracting drift in ion-selective field effect transistors (ISFETs). IEEE Sens. J. 2004, 4, 795-801.

(C) 2009 by the authors; licensee Molecular Diversity Preservation International, Basel, Switzerland. This article is an open-access article distributed under the terms and conditions of the Creative Commons Attribution license (http://creativecommons.org/licenses/by/3.0/). 